\title{
Analysis of paternal transmission of mitochondrial DNA in Drosophila
}

\author{
Wushur Sherengul ${ }^{1}$, Rumi Kondo ${ }^{2}$ and Etsuko T. Matsuura ${ }^{2 *}$ \\ ${ }^{1}$ Department of Advanced Biosciences, Ochanomizu University, Otsuka 2-1-1, \\ Bunkyo-ku, Tokyo 112-8610, Japan \\ ${ }^{2}$ Department of Biology, Ochanomizu University, Otsuka 2-1-1, \\ Bunkyo-ku, Tokyo 112-8610, Japan
}

(Received 28 September 2006, accepted 26 November 2006)

\begin{abstract}
It has previously been shown that paternal mitochondrial DNA (mtDNA) can be detected in later generations in Drosophila. To further analyze the paternal transmission of mtDNA, the progeny of two intraspecific and three interspecific crosses were examined in the frequency of the paternal transmission of mtDNA, using closely related species of the melanogaster species subgroup. Types of mtDNA in the progeny of the individual backcrosses of $F_{1}$ females were analyzed by selective amplification of paternal mtDNA. More than $100 \mathrm{~F}_{1}$ females were examined for each backcross. The same type of mtDNA as that of the paternal mtDNA was detected in approximately $20-60 \%$ of the backcrosses. The present results indicate that paternal leakage occurs in the intraspecific crosses as well as in the interspecific crosses in Drosophila.
\end{abstract}

Key words: Drosophila, mitochondrial DNA, paternal leakage

\section{INTRODUCTION}

Mitochondria have their own genomes in multiple copies and show different modes of inheritance from the nuclear genome. Mitochondrial DNA inheritance has been believed to be strictly maternal (e.g., Giles et al., 1980; Dawid and Blackler, 1972). Increasingly, however, evidence from both natural population surveys and experimental crosses has suggested that paternal leakage and biparental inheritance of mtDNA may be more common. These examples include several groups of animals: insects (Drosophila, Kondo et al., 1990, 1992), mussels (Mytilus, Zouros et al., 1994), birds (Parus, Kvist et al., 2003), and mice (Gyllensten et al., 1991). It has been summarized that similar paternal leakage occurs in other groups of organisms (Xu, 2005). In humans and chimpanzees, recombination in mtDNA suggested from linkage disequilibrium study has been interpreted under the presence of paternal mtDNA (Awadalla et al., 1999). Theoretical population genetics studies have suggested that transmission of even a small amount of paternal mtDNA provides a sufficient extent of gene flow between isolated female lineages (Takahata and Maruyama, 1981; Chapman et al., 1982; Birky, 1983).

Maternal transmission of mtDNA might be explained by the stochastic loss of paternal mtDNA in a zygote

\section{Edited by Yoko Satta}

* Corresponding author. E-mail: etmatsu@cc.ocha.ac.jp
(Birky, 1983). In animals, a mature sperm carries approximately 100 copies of mtDNA (Hecht et al., 1984), in contrast, an oocyte contains $10^{5}$ to $10^{8}$ copies of mtDNA (Giles et al., 1980; Jansen and de Boer, 1998). In two inbred strains of $M$. musculus and $M$. spretus whose mtDNAs can easily be distinguished by the polymerase chain reaction (PCR), Gyllensten et al. (1991) have shown that paternally inherited mtDNA can be detected at a frequency of $10^{-4}$ relative to the maternal contributions. This amount of paternal mtDNA was not detected by restriction analysis in their early study (Gyllensten et al., 1985).

Another possibility is that a specific mechanism exists to distinguish sperm mtDNA from maternal mtDNA in a zygote. Paternal transmission has been reported in interspecific rather than intraspecific hybrids (Kondo et al., 1990; Gyllensten et al., 1991). Kaneda et al. (1995) have indicated that paternal mtDNA can be detected in neonates only in the case of an interspecific cross in mice, between $M$. musculus and $M$. spretus, and have suggested active degradation of sperm mitochondria by loss of their membrane potential at the early stage of embryos. Later, the loss of paternal mitochondria was also shown to be tissue-specific by microinjection experiments in mouse embryos (Shitara et al., 2000). That is, when mitochondria from liver or spermatid were injected into zygotes, the exogenous mtDNA from spermatid was not detected in the progeny, but mtDNA from liver was detected. However, molecular mechanisms for the recognition of sperm mitochondria have not yet been clarified. 
Drosophila provides a useful system for the study of paternal transmission of mtDNA by the availability of many closely related species. Evidence of paternal mtDNA transmission was also found in Drosophila. Sperm mtDNA was detected in the progeny of backcross lines after ten consecutive interspecific backcrosses, $D$. simulans (female) $\times D$. mauritiana (male) (Kondo et al., 1990). In this experiment, Southern hybridization was used to detect paternal mtDNA in amounts at least $0.03 \%$ of a mixture with maternal mtDNA. Afterwards, PCR techniques were applied to detect paternal mtDNA in the presence of a $10^{3}$-fold excess of highly homologous maternal DNA. Paternal mtDNA was detected in the progeny of both one intraspecific cross in $D$. simulans and one interspecific cross between $D$. simulans (female) $\times D$. mauritiana (male) (Kondo et al., 1992). These results are in contrast to those in mice indicating that paternal mtDNA can be detected only in interspecific crosses. The reason for this inconsistency has not yet been clarified. More extensive studies are necessary to investigate the differences between mice and Drosophila, which would be of some help to understanding the mode of paternal inheritance of mtDNA.

In the present study, we examined the presence of paternal mtDNA in the progeny of two intraspecific crosses within $D$. simulans and D. mauritiana, and three interspecific crosses among $D$. simulans, $D$. mauritiana and $D$. sechellia, by designing the 3 ' end of primers of PCR to selectively amplify a fragment derived from paternal mtDNA. The results indicated detection of paternal mtDNA in approximately $20-60 \%$ of the backcrosses from all the combinations examined.

\section{MATERIALS AND METHODS}

Strains and crosses Three of the closely related spe- cies of the melanogaster species subgroup were used: $D$. simulans (NOU1, SL61, and RE213 strains represent siI, siII, and siIII mtDNA type, respectively), D. mauritiana (g20 and g52 strains represent $m a \mathrm{I}$ and $m a \mathrm{II}$ mtDNA type, respectively), and D. sechellia (SS51 strain). MtDNA of these types can be easily distinguished by their restriction fragments by HpaII. When these three species are hybridized by each other reciprocally, the male progeny from interspecific crosses is sterile, except for the rare fertile male progeny from a cross between $D$. mauritiana (female) and D. simulans (male) (Lemeunier et al., 1986). For a cross, three virgin females were mated with more than five males at $25^{\circ} \mathrm{C}$. After eggs were laid, all the parental individuals were discarded. Due to the availability of the 3' end of the PCR primers described below, two intraspecific crosses and three interspecific crosses were made: $s i \mathrm{III}$ (female) $\times$ siI (male) and maII (female) $\times m a \mathrm{I}$ (male) for the intraspecific combinations, and $s i \mathrm{I}$ (female) $\times m a \mathrm{I}$ (male), sec (female) $\times$ siII (male), and $m a \mathrm{II}$ (female) $\times$ siI (male) for the interspecific combinations. We randomly selected virgin $F_{1}$ females and individually backcrossed to 3 to 5 males of its maternal and paternal strains, respectively. Then mtDNA was extracted from approximately 50 adult flies of the progeny of each $F_{1}$ female as described below. We tested more than $100 \mathrm{~F}_{1}$ females in each backcross.

PCR analysis We designed primers to discriminate paternal from maternal mtDNA, based on the method of Kondo et al. (1992). To design primers for each cross, we first searched the entire sequences of the coding region in mtDNA for these species in the database, and selected sequences as primers that were perfectly matched at their 5 ' ends but differed at the 3 ' ends. In addition, primers were selected so as to amplify the DNA segment containing a restriction enzyme cleavage site for easy confirma-

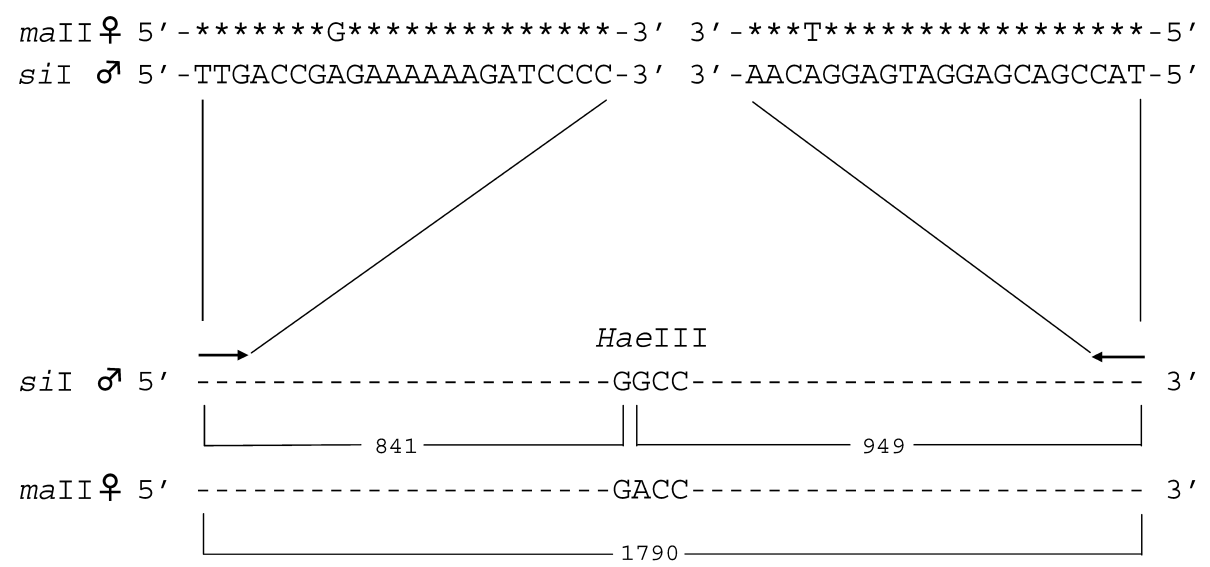

Fig. 1. PCR primers and the expected size of the amplified fragment after HaeIII digestion for a cross of maII females and siI males. Asterisks in the maII sequence indicate the sequence identity with siI mtDNA. Sequences of siI mtDNA were used for the primers. The sizes of the region amplified and fragments cut by HaeIII are indicated. 
Table 1. Primers and restriction enzymes used in the present experiments

\begin{tabular}{|c|c|c|c|c|c|}
\hline & 우 & $\delta$ & $\begin{array}{l}\text { PCR product } \\
\text { (bp) }\end{array}$ & Primers & $\begin{array}{l}\text { Restriction } \\
\text { enzyme }\end{array}$ \\
\hline \multirow{2}{*}{$\begin{array}{l}\text { Intraspecific } \\
\text { crosses }\end{array}$} & $m a \mathrm{II}$ & $m a \mathrm{I}$ & 3337 & $\begin{array}{l}\text { CTCAACTTTCTTATTCCCCAGC } \\
\text { TCGAGATGAAGATTTTGGGTC }\end{array}$ & EcoRI \\
\hline & siIII & $s i \mathrm{I}$ & 1799 & $\begin{array}{l}\text { TTGACCGAGAAAAAAGATCCC } \\
\text { TGGCTGCTCCTACTCCTGTT }\end{array}$ & HaeIII \\
\hline \multirow{3}{*}{$\begin{array}{l}\text { Interspecific } \\
\text { crosses }\end{array}$} & $m a \mathrm{II}$ & $s i \mathrm{I}$ & 1790 & $\begin{array}{l}\text { TTGACCGAGAAAAAAGATCCCC } \\
\text { ATGGCTGCTCCTACTCCTGTT }\end{array}$ & HaeIII \\
\hline & $s i \mathrm{I}$ & $m a \mathrm{I}$ & 5044 & $\begin{array}{l}\text { CGACGATATTCAGATTACCCAG } \\
\text { GGGTGAGATGGATTAGGACT }\end{array}$ & HindIII \\
\hline & $s e c$ & siII & 2282 & $\begin{array}{l}\text { CCTGATATAGCATTCCCGCG } \\
\text { TTAGGTGAATCTGGCATGTAAG }\end{array}$ & HpaI \\
\hline
\end{tabular}

tion of the sequence. Amplified mtDNA of a paternal strain was cleaved by a restriction enzyme, but mtDNA of the maternal strain was not digested by the same restriction enzyme. An example of the cross between the maII female and siI male is shown in Fig. 1. The oligonucleotide primers and restriction enzymes used are summarized in Table 1 . These PCR amplified fragments were sequenced, and all the restriction sites were confirmed.

To confirm the limit of the detection of paternal mtDNA, PCR was carried out using templates mixed maternal mtDNA with paternal mtDNA at various ratios from $10^{-3}$ to $10^{-9}$ for each cross tested.

MtDNA was extracted through SDS-phenol treatment of mitochondria isolated from homogenates of adult flies by differential centrifugation (Satta et al., 1987) and used as templates for the PCR. The reaction was performed using a DNA thermal cycler (TaKaRa PCR Thermal Cycler PERSONAL) in a total $50 \mu \mathrm{l}$ of a solution containing $0.5 \mu \mathrm{M}$ each of primers, $5 \mu \mathrm{l}$ of $10 \times$ buffer, $1.5 \mathrm{mM}$ $\mathrm{MgCl}_{2}, 200 \mu \mathrm{M}$ dNTP, $25 \mathrm{ng}$ of template mtDNA, and 2.5 units of rTaq polymerase. Each PCR product was then treated with a suitable restriction enzyme and applied on agarose gels for confirmation of the paternal-specific restriction site.

\section{RESULTS}

Based on the method using designed primers that discriminate paternal from maternal mtDNA, we examined the presence of paternal mtDNA in the progeny of two intraspecific crosses, $m a \mathrm{II}$ (female) $\times m a \mathrm{I}$ (male) and siIII (female) $\times s i \mathrm{I}$ (male), and three interspecific crosses, si I (female) $\times$ maI (male), sec (female $) \times$ siII (male), and maII (female) $\times$ siI (male).

First, we examined the sensitivity of the detection by a series of PCR for each cross. Approximately $25 \mathrm{ng}$ of the template DNA was used, which contained mtDNA from a maternal strain mixed with that from a paternal at various ratios; $1: 1,1: 10^{-2}, 1: 10^{-3}, 1: 10^{-4}, 1: 10^{-5}, 1: 10^{-6}, 1: 10^{-7}$, $1: 10^{-8}$, and $1: 10^{-9}$. Fig. 2 shows an example of the results of PCR for such control experiments for the combination (a)

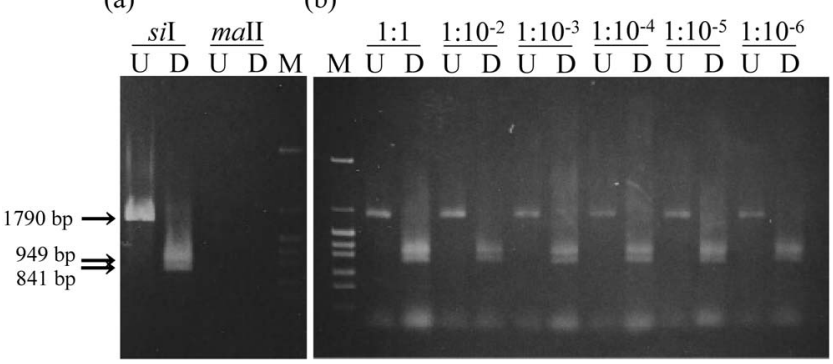

Fig. 2. An example of the results of PCR to determine the limit for the detection of paternal mtDNA. maII mtDNA and siI mtDNA were used as the maternal and paternal mtDNA, respectively. PCR products that were undigested (U) and digested (D) by HaeIII are shown for each reaction. Lane $\mathrm{M}$ is the pHY molecular weight marker. (a) Only siI mtDNA or maII mtDNA was used as templates. (b) maII mtDNA and siI mtDNA were mixed at various ratios. The ratios $m a \mathrm{II}: s i \mathrm{I}$ are indicated above the lanes.

of maII (female) and siI (male) mtDNA. When either maII or siI mtDNA was used as templates, amplification was observed only from siI mtDNA. For the mixture of the two kinds of mtDNA, paternal siI mtDNA could be detected at $10^{-6}$, but it was not detected at $10^{-7}$. The PCR amplified fragment from siI was $1790 \mathrm{bp}$, and after cleavage by a paternal-specific restriction enzyme HaeIII, two fragments, $949 \mathrm{bp}$ and $841 \mathrm{bp}$, were detected. These two fragments indicated the presence of the paternal mtDNA.

Paternal mtDNA was detected at a frequency of $10^{-3}$ $10^{-6}$, depending on the cross tested. It was observed that the sensitivity of the primer set tended to be higher for the interspecific combinations than for the intraspecific combinations. The sensitivity was the lowest, $10^{-3}$, for the combination of maII (female) and maI (male) mtDNA, and the highest, $10^{-6}$, for the combination of maII (female) and siI (male) mtDNA and siI (female) and maI (male) mtDNA.

Table 2 summarizes the results of the detection of paternal mtDNA for the intra- and interspecific crosses. The $F_{1}$ females were individually backcrossed to males of a paternal and a maternal strain, respectively. For one 
Table 2. Proportions of the backcross of $\mathrm{F}_{1}$ individual females that detected paternal mtDNA

\begin{tabular}{lcccc}
\hline \hline & & \multicolumn{2}{c}{ Males backcrossed to $\mathrm{F}_{1}$ females } \\
\cline { 3 - 5 } & \multirow{2}{*}{} & $\delta$ & Paternal strain & Maternal strain \\
\hline Intraspecific & $m a \mathrm{II}$ & $m a \mathrm{I}$ & $22 / 103(21.3 \%)$ & $19 / 102(18.6 \%)$ \\
crosses & $s i \mathrm{III}$ & $s i \mathrm{I}$ & $52 / 163(31.9 \%)$ & $63 / 130(48.4 \%)$ \\
\hline \multirow{2}{*}{ Interspecific } & $m a \mathrm{II}$ & $s i \mathrm{I}$ & $86 / 151(56.9 \%)$ & $50 / 109(45.8 \%)$ \\
crosses & $s i \mathrm{I}$ & $m a \mathrm{I}$ & $72 / 114(63.1 \%)$ & $52 / 135(38.5 \%)$ \\
& $s e c$ & $s i \mathrm{II}$ & $36 / 103(34.9 \%)$ & $34 / 111(30.6 \%)$ \\
\hline
\end{tabular}

Numbers indicate the number of backcrosses that detected paternal mtDNA / the total number of backcrosses tested.

of the intraspecific crosses, $m a \mathrm{II}($ female $) \times m a \mathrm{I}$ (male), a total of $103 \mathrm{~F}_{1}$ individuals were examined by their progeny of the backcrosses to males of a paternal strain, and the paternal mtDNA type was detected in 22 backcrosses (21.3\%). In contrast, in the cases of $102 \mathrm{~F}_{1}$ backcrossed to males of a maternal strain, paternal mtDNA was detected in 19 backcrosses (18.6\%). The difference in the frequencies of paternal leakage was not statistically significant $\left(\chi^{2}=0.24, P>0.05\right)$. In the other combination of $s i$ III (female) $\times$ siI (male), the paternal mtDNA type was also detected in the progeny of the $F_{1}$, backcrossed to both a maternal and a paternal strain. However, the frequency of paternal leakage in the backcross to a maternal strain was significantly higher than that in the backcross to a paternal strain $\left(\chi^{2}=8.31, P<0.01\right)$. The total frequencies of the presence of paternal mtDNA among the progeny from the two kinds of backcrosses were $28 \%$ and $35 \%$ for a paternal and a maternal strain, respectively. This difference was not statistically significant $\left(\chi^{2}=3.26, P>0.05\right)$.

In the three interspecific combinations, paternal mtDNA was also detected in all the crosses. In maII $($ female $) \times s i I$ (male), for example, the paternal mtDNA was detected in 86 of 151 backcrosses of the $F_{1}$ females to males of a paternal strain. When the $F_{1}$ females were backcrossed to males of a maternal strain, paternal mtDNA was detected in 50 of 109 backcrosses. The total frequency of the presence of paternal mtDNA among the progeny was significantly higher in backcrosses to a paternal strain, $53 \%$, than in backcrosses to a maternal strain, $38 \%\left(\chi^{2}=15.11, P<0.01\right)$.

\section{DISCUSSION}

In the present study, using three species belonging to the melanogaster species subgroup of Drosophila, the transmission of paternal mtDNA was investigated by PCR, using mtDNA extracted from their backcross progeny for both intra- and interspecific crosses. The results showed that paternal mtDNA was detected in approximately $20-60 \%$ of the backcrosses.

It has been known that nuclear DNA homologous to mtDNA, which is called nuclear mtDNA (NUMT), exists (Lopez et al., 1994). According to Richly and Leister
(2004), less than ten NUMTs were found in Drosophila, and a total amount of them was about only $500 \mathrm{bp}$. They also described that NUMTs showed variable degrees of homology to mtDNA and that rearrangement and fragmentation of mtDNA sequences were observed. In the present study, five different kinds of PCR were performed to amplify mtDNA sequences that were more than $1.8 \mathrm{~kb}$ long. In addition, the primer sequences used were very carefully designed so as to selectively amplify paternal mtDNA in the presence of highly homologous maternal mtDNA. Therefore, the possibility to amplify NUMTs by these primers could be excluded.

Only paternal mtDNA incorporated into germ cells of the $F_{1}$ females was transmitted to the progeny of the backcrosses. Therefore, the higher frequencies in backcrosses to a paternal strain in most cases might indicate that some paternal mtDNA was further incorporated at the backcross and remained in somatic cells. The reason for the lower frequency in a backcross to a paternal strain observed in the case of siIII (female) $\times$ siI (male) is unclear at present. It is noted that the transmission of paternal mtDNA through germ cells, which was observed in the backcrosses to males of a maternal strain, was detected for the intraspecific crosses as well as for the interspecific crosses in Drosophila. In addition, the frequencies in the intraspecific crosses might be underestimated due to lower sensitivities of the primer sets of PCR, as compared to the interspecific crosses.

In mice, paternal mtDNA was shown to be eliminated at the late pronucleus stage of intraspecific hybrid embryos. The leaked paternal mtDNA was not distributed to all tissues of the interspecific $F_{1}$ hybrid and was also limited to the first generation in a cross of M. musculus and $M$. spretus (Shitara et al., 1998). In another study in mice, in the intraspecific hybrids in M. musculus, paternal mtDNA was detected only through the early pronuclear stage (Kaneda et al., 1995). They concluded that cytoplasmic genomes were transmitted uniparentally only in intraspecific crosses. It is likely that the mechanisms that recognize sperm mitochondria or mtDNA of the same species should be ineffective against foreign mitochondria or mtDNA derived from a different species, since interspecific hybridization does not usually occur in natural populations. As compared with the cases in mice, the 
present results indicate that paternal leakage is not prohibited even in intraspecific crosses in Drosophila, and confirm the results of previous studies (Kondo et al., 1990, 1992). Although the differences in the frequencies of paternal leakage between the intraspecific and interspecific crosses were not clear, the present results may suggest that the recognition mechanism of sperm mitochondria in Drosophila would be different from that in mice. For further investigation, the determination of the amount of paternal mtDNA in individual flies would be indispensable.

A number of studies have indicated that sperm mitochondria are actively destroyed in egg (Hiraoka and Hirao, 1988; Shalgi et al., 1994; Sutovsky et al., 1996, 2000). It has been indicated that sperm mitochondria are ubiquitinated during spermatogenesis in bovine, rhesus, mice, and human (Sutovsky et al., 2000). Ubiquitination is a universal marker of proteolysis and protein recycling, showing a high degree of evolutionary conservation (Sutovsky et al., 2000). Clarification of the extent of ubiquitination of sperm in Drosophila would be of some help in understanding the mechanisms of paternal leakage. On the other hand, the mitochondria in sperm have been known to undergo morphological changes during spermatogenesis in insects. They aggregate and fuse to form one or two derivatives that run longitudinally along one side of the axoneme for nearly the full length of the sperm tail (Fuller, 1993). After the entry of one sperm into an egg cell from the anterior end, the sperm were observed to show species-specific folding and coiling patterns in eggs (Pitnick et al., 1995; Pitnick and Karr, 1998). For paternal leakage to occur, sperm mitochondria or derivatives should be incorporated into pole cells formed at the posterior end. More careful observation is necessary to understand the sperm activity in a fertilized egg.

The present study suggests that sperm mtDNA is transmitted in Drosophila, which might possess different recognition mechanisms from those in mammals. Further qualitative and quantitative studies are required to assess the evolutionary diversity of the modes and effects of paternal leakage in animals.

\section{REFERENCES}

Awadalla, P., Eyre-Walker, A., and Smith, J. M. (1999) Linkage disequilibrium and recombination in hominoid mitochondrial DNA. Science 286, 2524-2525.

Birky, C. W., Jr. (1983) Relaxed cellular controls and organelle heredity. Science 222, 468-475.

Chapman, R. W., Stephens, J. C., Lansman, R. A., and Avise, J. C. (1982) Models of mitochondrial DNA transmission genetics and evolution in higher eukaryotes. Genet. Res., Camb. 40, 41-57.

Dawid, I. B., and Blackler, A. W. (1972) Maternal and cytoplasmic inheritance of mitochondrial DNA in Xenopus. Dev.
Biol. 29, 152-161.

Fuller, M. T. (1993) Spermatogenesis. In: The Development of Drosophila melanogaster (eds.: M. Bate, and A. M. Arias), pp. 71-147. Cold Spring Harbor Laboratory Press.

Giles, R. E., Blanc, H., Cann, H. M., and Wallace, D. C. (1980) Maternal inheritance of human mitochondrial DNA. Proc. Natl. Acad. Sci. USA 77, 6715-6719.

Gyllensten, U., Wharton, D., and Wilson, A. C. (1985) Maternal inheritance of mitochondrial DNA during backcrossing of two species of mice. J. Hered. 76, 321-324.

Gyllensten, U., Wharton, D., Josefsson, A., and Wilson, A. C. (1991) Paternal inheritance of mitochondrial DNA in mice. Nature 352, 255-257.

Hecht, N. B., Liem, H., Kleene, K. C., Distel, R. J., and Ho, S. M. (1984) Maternal inheritance of the mouse mitochondrial genome is not mediated by a loss or gross alteration of the paternal mitochondrial DNA or by methylation of the oocyte mitochondrial DNA. Dev. Biol. 102, 452-461.

Hiraoka, J., and Hirao, Y. (1988) Fate of sperm tail components after incorporation into the hamster egg. Gamete Res. 19 369-380.

Jansen, R. P., and de Boer, K. (1998) The bottleneck: mitochondrial imperatives in oogenesis and ovarian follicular fate. Mol. Cell. Endocrinol. 145, 81-88.

Kaneda, H., Hayashi, J.-I., Takahama, S., Taya, C., Lindahl, K. F., and Yonekawa, H. (1995) Elimination of paternal mitochondrial DNA in intraspecific crosses during early mouse embryogenesis. Proc. Natl. Acad. Sci. USA 92, 4542-4546.

Kondo, R., Satta, Y., Matsuura, E. T., Ishiwa, H., Takahata, N., and Chigusa, S. I. (1990) Incomplete maternal transmission of mitochondrial DNA in Drosophila. Genetics 126, 657663.

Kondo, R., Matsuura, E. T., and Chigusa, S. I. (1992) Further observation of paternal transmission of Drosophila mitochondrial DNA by PCR selective amplification method. Genet. Res., Camb. 59, 81-84.

Kvist, L., Martens, J., Nazarenko, A. A., and Orell, M. (2003) Paternal leakage of mitochondrial DNA in the great tit (Parus major). Mol. Biol. Evol. 20, 243-247.

Lemeunier, F., David, J. R., and Tsacas, L. (1986) The melanogaster species group. In: The Genetics and Biology of Drosophila, Vol. 3e. (eds.: M. Ashuburner, H. L. Carson, and J. N. Thompson, Jr.), pp. 147-256. Academic Press, London.

Lopez, J. V., Yuhki, N., Masuda, R., Modi, W., and O’Brien, S. J. (1994) Numt, a recent transfer and tandem amplification of mitochondrial DNA to the nuclear genome of the domestic cat. J. Mol. Evol. 39, 174-190.

Pitnick, S., and Karr, T. L. (1998) Paternal products and byproducts in Drosophila development. Proc. R. Soc. Lond. B 265, 821-826.

Pitnick, S., Markow, T. A., and Spicer, G. S. (1995) Delayed male maturity is a cost of producing large sperm in Drosophila. Proc. Natl. Acad. Sci. USA 92, 10614-10618.

Richly, E., and Leister, D. (2004) NUMTs in sequenced eukaryotic genomes. Mol. Biol. Evol. 21, 1081-1084.

Satta, Y., Ishiwa, H., and Chigusa, S. I. (1987) Analysis of nucleotide substitutions of mitochondrial DNAs in Drosophila melanogaster and its sibling species. Mol. Biol. Evol. 4, 638-650.

Shalgi, R., Magnus, A., Jones, R., and Phillips, D. M. (1994) Fate of sperm organelles during early embryogenesis in the rat. Mol. Reprod. Dev. 37, 264-271.

Shitara, H., Hayashi, J.-I., Takahama, S., Kaneda, H., and Yonekawa, H. (1998) Maternal inheritance of mouse 
mtDNA in interspecific hybrids: segregation of the leaked paternal mtDNA followed by the prevention of subsequent paternal leakage. Genetics 148, 851-857.

Shitara, H., Kaneda, H., Sato, A., Inoue, K., Ogura, A., Yonekawa, H., and Hayashi, J.-I. (2000) Selective and continuous elimination of mitochondria microinjected into mouse eggs from spermatids, but not from liver cells, occurs throughout embryogenesis. Genetics 156, 1277-1284.

Sutovsky, P., Navara, C. S., and Schatten, G. (1996) Fate of the sperm mitochondria, and the incorporation, conversion and disassembly of the sperm tail structures during bovine fertilization. Biol. Reprod. 55, 1195-1205.

Sutovsky, P., Moreno, R. D., Ramalho-Santos, J., Dominko, T.,
Simerly, C., and Schatten, G. (2000) Ubiquitinated sperm mitochondria, selective proteolysis, and the regulation of mitochondrial inheritance in mammalian embryos. Biol. Reprod. 63, 582-590.

Takahata, N., and Maruyama, T. (1981) A mathematical model of extranuclear genes and the genetic variability maintained in a finite population. Genet. Res., Camb. 37, 291-302.

$\mathrm{Xu}$, J. (2005) The inheritance of organelle genes and genomes: patterns and mechanisms. Genome 48, 951-958.

Zouros, E., Oberhauser B. A., Saavedra, C., and Freeman, K. R. (1994) An unusual type of mitochondrial DNA inheritance in the blue mussel Mytilus. Proc. Natl. Acad. Sci. USA 91, 7463-7467. 